\title{
TOPOLOGICAL ENTROPY BOUNDS MEASURE-THEORETIC ENTROPY
}

\section{WAYNE GOODWYN}

Let $T$ be a homeomorphism from a compact space $X$ onto itself and let $\mu$ be a $T$-invariant probability measure on the Borel sets of $X$. It was conjectured in [1] that the measure-theoretic entropy of $T$ with respect to $\mu$ is less than or equal to the topological entropy of $T$. The purpose of this paper is to show, under the assumption that $X$ is metric, that the inequality holds when $T$ is assumed only to be a continuous map from $X$ into itself.

We shall first prove the inequality under the assumption that $X$ is a closed subset of the Hilbert cube which is invaraint under a certain type of shift operator, and $T$ is the restriction of the operator to $X$. The generalization will be obtained by considering representations of $T$ as such shifts.

By a flow we mean a pair $(X, T)$, where $X$ is a compact metric space and $T$ is a continuous map from $X$ into itself. Throughout the paper, $(X, T)$ and $(Y, S)$ will denote arbitrary flows. A continuous map $\phi: X \rightarrow Y$ will be called a homomorphism from $(X, T)$ into $(Y, S)$ if $\phi \circ T=S \circ \phi$. If $\alpha$ is any finite cover of $X$, we let $N(\alpha)$ be the number of members in a subcover of $\alpha$ of minimal cardinality. As in [1], we write $\alpha \bigvee \beta=\{U \cap V: U \in \alpha, V \in \beta\}$ and we write $\alpha>\beta$ to mean that $\alpha$ is a refinement of $\beta$, though this is contrary to the notation of many authors. As in [1], it follows from the fact that $N(\alpha \vee \beta)$ $\leqq N(\alpha) \cdot N(\beta)$, that the limit exists in the following definition:

$$
h(\alpha, T)=\lim _{n \rightarrow \infty} \frac{1}{n} \log N\left(\bigvee_{i=0}^{n-1} T^{-i} \alpha\right),
$$

for any finite cover $\alpha$ of $X$. Finally, we note that if $\alpha>\beta$, then $N(\alpha)$ $\geqq N(\beta)$, and $h(\alpha, T) \geqq h(\beta, T)$. The topological entropy of $T$ is defined as

$$
h(T)=\sup h(\alpha, T),
$$

where the supremum is taken over all finite open covers of $X$.

It is easily seen that if $\phi$ is a homomorphism from $(X, T)$ onto $(Y, S)$ and if $\alpha$ is a finite cover of $Y$, then $h\left(\phi^{-1}(\alpha), T\right)=h(\alpha, S)$. It follows that $h(S) \leqq h(T)$.

Received by the editors December 16, 1968. 
Let $M(X, T)$ denote the set of all $T$-invariant probability measures on the Borel sets of $X$. For a finite measurable partition $\alpha$ of $X$, we write, as in [2],

$$
H_{\mu}(\alpha)=-\sum_{A \in \alpha} \mu(A) \log \mu(A),
$$

and

$$
h_{\mu}(\alpha, T)=\lim _{n \rightarrow \infty} \frac{1}{n} H_{\mu}\left(\bigvee_{i=0}^{n-1} T^{-i} \alpha\right) .
$$

The measure-theoretic entropy of $T$ is defined as

$$
h_{\mu}(T)=\sup h_{\mu}(\alpha, T),
$$

where the supremum is taken over all finite measurable partitions of $X$.

For every cover $\alpha$ of $X$, we write

$$
w(\alpha)=U\{U \cap V: U, V \in \alpha ; U \neq V\} .
$$

If $\mu$ is a measure on the Borel sets of $X$, we say that a cover $\alpha$ is $\mu$ disjoint whenever $\mu(w(\alpha))=0$. For $\mu \in M(X, T)$, it is easy to show that if $\alpha$ is $\mu$-disjoint then so is $\bigvee_{i=0}^{n-1} T^{-i} \alpha$. It follows that $h_{\mu}(\alpha, T)$ is defined for any finite measurable $\mu$-disjoint cover $\alpha$ of $X$.

By an essential member of a cover $\alpha$ we mean a member $U$ such that $\alpha-\{U\}$ is not a cover.

Proposition 1. Let $\mu \in M(X, T)$ and let $\alpha$ be a finite measurable $\mu$-disjoint cover of $X$. Then

$$
h_{\mu}(\alpha, T) \leqq h(\alpha, T) .
$$

Proof. Fix a positive integer $n$ and let $\beta$ be the set of members of $\bigvee_{i=0}^{n-1} T^{-i} \alpha$ which have positive measure. Let $k$ be the number of members of $\beta$. It follows from the fact that $\bigvee_{i=0}^{n-1} T^{-i} \alpha$ is $\mu$-disjoint that each set of $\beta$ is essential to $\bigvee_{i=0}^{n-1} T^{-i} \alpha$, so that $k \leqq N\left(\bigvee_{i=0}^{n-1} T^{-i} \alpha\right)$. On the other hand, it follows from the convexity of the function $t \log t$ that $H_{\mu}(\beta) \leqq \log k$. (See $[4$, p. 4].) We can conclude that

$$
H_{\mu}\left(\bigvee_{i=0}^{n-1} T^{-i} \beta\right)=H_{\mu}(\beta) \leqq \log N\left(\bigvee_{i=0}^{n-1} T^{-i} \alpha\right) \text {. }
$$

We now divide by $n$ and let $n$ tend to infinity, obtaining the result.

To make use of Proposition 1 we must be able to compare $h(\alpha, T)$ with $h(T)$ for a $\mu$-disjoint cover $\alpha$ of $X$. We let $p(\alpha)$ denote the order of $\alpha$, the largest number of distinct members of $\alpha$ with a nonempty intersection. 
Proposition 2. If $\alpha$ is a finite closed cover of $X$, then

$$
h(\alpha, T) \leqq h(T)+\log p(\alpha) .
$$

Proof. For $x \in X$, let $\operatorname{St}(\alpha, x)$ denote the union of the members of $\alpha$ which contain $x$, and let $\operatorname{St}(\alpha)=\{\operatorname{St}(\alpha, x): x \in X\}$. Let $n$ be a positive integer. We claim that

$$
N\left(\bigvee_{i=0}^{n-1} T^{-i} \alpha\right) \leqq N\left(\bigvee_{i=0}^{n-1} T^{-i} \operatorname{St}(\alpha)\right) \cdot p(\alpha)^{n}
$$

For let $\gamma$ be a subcover of $\mathrm{V}_{i=0}^{n-1} T^{-i} \mathrm{St}(\alpha)$ of minimal cardinality. Then each member $F$ of $\gamma$ is of the form

$$
\begin{aligned}
& F=F_{0} \cap T^{-1} F_{1} \cap \cdots \cap T^{-n+1} F_{n-1}, \\
& \text { where } F_{i} \in \operatorname{St}(\alpha), \quad i=0, \cdots, n-1 .
\end{aligned}
$$

Now each $F_{i}$ is a union of at most $p(\alpha)$ members of $\alpha$, so $F$ is a union of at most $p(\alpha)^{n}$ members of $\bigvee_{i=0}^{n-1} T^{-i} \alpha$. Hence, from $\gamma$ we obtain a subcover of $\bigvee_{i=0}^{n=1} T^{-i} \alpha$ with at most

$$
N\left(\bigvee_{i=0}^{n-1} T^{-i} \mathrm{St}(\alpha)\right) \cdot p(\alpha)^{n}
$$

members. This proves the above inequality. If we now take the logarithm of both sides of the inequality, divide by $n$ and let $n$ tend to infinity, we obtain

$$
h(\alpha, T) \leqq h(\operatorname{St}(\alpha), T)+\log p(\alpha) .
$$

We next claim that for each $x \in X, x$ is an interior point of $\operatorname{St}(\alpha, x)$. This follows from the fact that the intersection of the complements of the sets of $\alpha$ which do not contain $x$ is an open subset of $\operatorname{St}(\alpha, x)$. It now follows that $\operatorname{St}(\alpha)$ has an open refinement, so that $h(\operatorname{St}(\alpha), T)$ $\leqq h(T)$, and the proposition is proved.

It should be remarked that a finite closed cover can yield entropy strictly greater than the topological entropy. (See $[3$, p. 45].)

The following theorem will be used to obtain $h_{\mu}(T)$ in terms of the entropy of finite, $\mu$-disjoint closed covers of $X$. The theorem is due to Rohlin, [4]; it appears in this form in [2, p. 87].

TheOREM. Let $\mu \in M(X, T)$, and let $\alpha_{0}<\alpha_{1}<\cdots$ be a sequence of finite $\mu$-disjoint measurable covers of $X$ such that the smallest sigma- 
algebra containing $\bigcup_{i=1}^{\infty} \bigcup_{k=1}^{\infty} T^{-i} \alpha_{k}$ is the algebra of all Borel sets of $X$. Then

$$
h_{\mu}(T)=\lim _{k \rightarrow \infty} h_{\mu}\left(\alpha_{k}, T\right) .
$$

Our next step will be to show that for certain subsets of the Hilbert cube we can obtain a sequence $\alpha_{0}<\alpha_{1}<\cdots$ of covers to which we can apply Propositions 1 and 2, and Rohlin's Theorem.

Throughout the paper, $Z^{+}$will denote the set of nonnegative integers.

Let $I^{n}$ denote the $n$-cube,

$$
I^{n}=\left\{u=\left(u_{0}, u_{1}, \cdots, u_{n-1}\right): 0 \leqq u_{i} \leqq 1 \text { for } i=0, \cdots, n-1\right\} .
$$

We shall find it useful to let $I^{n}$ have the metric $d$ defined as follows:

$$
d(u, v)=\max _{i=0, \cdots, n-1}\left|\mu_{i}-v_{i}\right| \quad \text { for } \mu, v \in I^{n} .
$$

For each positive integer $n$ we define $B_{n}$ to be the set of all sequences $x=(x(0), x(1) \cdots)$ of points in $I^{n}$; that is, $B_{n}=\left(I^{n}\right) Z^{+}$. We let $B_{n}$ have the metric $\rho$ defined as follows:

$$
\rho(x, y)=\sum_{m=0}^{\infty} 2^{-m} d(x(m), y(m)) .
$$

We let $\sigma_{n}: B_{n} \rightarrow B_{n}$ be the shift transformation on $B_{n}$ defined by: $\sigma_{n}(x)(m)=x(m+1)$ for $m \in N, x \in B_{n}$. Finally, we let $\pi_{n}: B_{n} \rightarrow I_{n}$ be the projection:

$$
\pi_{n}(x)=x(0) \quad \text { for } x \in B_{n} .
$$

Proposition 3. $\left(B_{n}, \sigma_{n}\right)$ is isomorphic to $\left(B_{1}, \sigma_{1}^{n}\right)$ for any positive integer $n$.

Proof. Define $f: B_{1} \rightarrow B_{n}$ as follows:

$$
(f(x)(m))_{i}=x(n m+i), \quad \text { for } 0 \leqq i<n, m \in Z^{+} .
$$

It is straightforward to show that $f$ is an isomorphism.

By mesh $(\alpha)$ we mean the supremum of the diameters of the sets in $\alpha$.

Proposition 4. Let $\alpha$ be a cover of $I^{n}$. If $m$ is a positive integer, then

$$
\operatorname{mesh}\left(\bigvee_{i=0}^{m-1} \sigma_{n}^{-i}\left(\pi_{n}^{-1}(\alpha)\right)\right) \leqq m \cdot \operatorname{mesh}(\alpha)+2^{-m+1} .
$$

The proof is straightforward. 
TheORem 1. Let $\nu$ be a finite measure on the Borel sets of $I^{n}$. Then there is a sequence $\beta_{0}<\beta_{1}<\cdots$ of closed $\nu$-disjoint covers of $I^{n}$ such that

(1) $\operatorname{mesh}\left(\beta_{i}\right) \rightarrow 0$.

(2) $p\left(\beta_{i}\right) \leqq n+1$ for $i \in Z^{+}$.

Before proving Theorem 1, we look at some corollaries. (Recall that $p\left(\beta_{i}\right)$ is the order of $\beta_{i}$.)

Corollary 1. Let $(X, T)$ be a subflow of $\left(B_{n}, \sigma_{n}\right)$; i.e., $X$ is a closed $\sigma_{n}$-invariant subset of $B_{n}$ and $T=\sigma_{n} \mid X$. Let $\mu \in M(X, T)$. Then $h_{\mu}(T) \leqq h(T)+\log (n+1)$.

Proof. Let $\beta_{0}<\beta_{1}<\cdots$ be a sequence obtained from Theorem 1 , where $\nu$ is defined by the rule

$$
\nu(A)=\mu\left(\pi_{n}^{-1}(A) \cap X\right) \quad \text { for every Borel set } A \text { of } I^{n} .
$$

Let $\alpha_{k}=\left\{\pi_{n}{ }^{-1}(F) \cap X: F \in \beta_{k}\right\}$, for $k \in N$. Then each $\alpha_{k}$ is a closed $\mu$-disjoint cover of $X$. We claim that $\left\{\alpha_{k}\right\}$ satisfies the hypothesis of Rohlin's Theorem. For let $x \in X$ and let $U$ be a neighborhood of $x$ in $X$. Choose $\epsilon>0$ so small that $\rho(x, y)<\epsilon$ implies $y \in U$, for $y \in X$. By Proposition 4 , we can choose $k, m \in Z^{+}$such that

$$
\operatorname{mesh}\left(\bigvee_{i=0}^{m-1} T^{-i} \alpha_{k}\right)<\epsilon \text {. }
$$

Hence, there is a set $F \in \bigvee_{i=0}^{m-1} T^{-i} \alpha_{k}$ such that $x \in F \subset U$. This shows that every open set is a union of sets in the countable collection $\bigcup_{j=0}^{\infty} \bigcup_{r=1}^{\infty} \mathrm{V}_{i=0}^{r-1} T^{-i} \alpha_{j}$. We now apply Rohlin's theorem and obtain $h_{\mu}(T)=\lim _{k \rightarrow \infty} h_{\mu}\left(\alpha_{k}, T\right)$. An application of Propositions 1 and 2 now completes the proof of the corollary.

Corollary 2. Let $(X, T)$ be a subflow of $\left(B_{n}, \sigma_{n}\right)$ and let $\mu \in M(X, T)$. Then $h_{\mu}(T) \leqq h(T)$.

Proof. Let $m$ be a positive integer. By Proposition $3,\left(X, T^{m}\right)$ is isomorphic to a subflow of $\left(B_{1}, \sigma_{1}^{n m}\right)$ which is isomorphic to $\left(B_{n m}, \sigma_{n m}\right)$. Hence, by Corollary $1, h_{\mu}\left(T^{m}\right)-h\left(T^{m}\right) \leqq \log (n m+1)$. Now recalling that $h_{\mu}\left(T^{m}\right)=m h_{\mu}(T)$ and $h\left(T^{m}\right)=m h(T)$, we have $h_{\mu}(T)-h(T)$ $=(1 / m)\left(h_{\mu}\left(T^{m}\right)-h\left(T^{m}\right)\right) \leqq(1 / m) \log (n m+1)$. We now observe that as $m$ tends to infinity, $(1 / m) \log (n m+1)$ tends to zero.

The proof of Theorem 1 proceeds by a series of lemmas. We let $\nu$ be a given finite measure on the Borel sets of $I^{n}$.

By an $n$-rectangle we mean a subset $R$ of $I^{n}$ of the form

$$
R=\left\{x=\left(x_{0}, \cdots, x_{n-1}\right) \in I^{n}: a_{i} \leqq x_{i} \leqq b_{i}, i=0, \cdots, n-1\right\},
$$


where $a_{i}, b_{i}$ are numbers such that $0 \leqq a_{i}<b_{i} \leqq 1$ for $i=0, \cdots n-1$. The $i$-mesh of $R$ is defined to be $b_{i}-a_{i}$.

By a rectangular cover of $I^{n}$ we mean a finite cover of $I^{n}$ consisting of $n$-rectangles. If $\alpha$ is a rectangular cover of $I^{n}$, we can write

$$
\begin{aligned}
& \alpha=\left\{R_{1}, \cdots, R_{q}\right\} \text {, where } \\
& R_{j}=\left\{x \in I^{n}: a_{i}^{j} \leqq x_{i} \leqq b_{i}^{j}, i=0, \cdots, n-1\right\} \\
& \text { for } j=1, \cdots, q \text {. }
\end{aligned}
$$

Definition. Let $\alpha$ be a rectangular cover of $I^{n}$ represented by $\left({ }^{*}\right)$. We define the $i$-mesh of $\alpha$ to be

$$
L_{i}(\alpha)=\max _{j=1, \cdots, q}\left(b_{i}^{j}-a_{i}^{j}\right) .
$$

Note that $\max _{i=0, \cdots n-1} L_{i}(\alpha)$ is the mesh of $\alpha$ with respect to the metric we defined on $I^{n}$.

Definition. Let $\alpha$ be a rectangular cover of $I^{n}$ represented by $\left(^{*}\right)$. For $x \in I^{n}$, we define

$$
\xi(\alpha, x)=\text { the number of integers } i \in\{0, \cdots, n-1\}
$$

such that there is a $j \in\{1, \cdots, q\}$ with $x_{i}=a_{i}^{j}$ or $x_{i}=b_{i}^{j}$. We say that $\alpha$ is uneven if

$$
p(\alpha, x) \leqq \xi(\alpha, x)+1 \quad \text { for all } x \in I^{n},
$$

where $p(\alpha, x)$ is the number of distinct members of $\alpha$ containing $x$.

LemMa 1. Let $\alpha$ be an uneven $\nu$-disjoint rectangular cover of $I^{n}$. Fix $R \in \alpha$ and $f x i \in\{0, \cdots n-1\}$. Then there is a decomposition of $R$,

$$
R=R^{\prime} \cup R^{\prime \prime} \text { such that } \alpha^{\prime}=\left\{R^{\prime}, R^{\prime \prime}\right\} \cup\{F \in \alpha: F \neq R\}
$$

is an uneven $\nu$-disjoint rectangular refinement of $\alpha$, and such that the $i$-mesh of $R^{\prime}$ and $R^{\prime \prime}$ are both $\leqq \frac{2}{3}$ the $i$-mesh of $R$.

Proof. Let $\alpha$ be represented by $\left({ }^{*}\right)$, where $R=R_{1}$. For $c \in\left(a_{i}^{1}, b_{i}^{1}\right)$ we define $H_{c}=\left\{x \in I^{n}: x_{i}=c\right\}$. We now choose

$$
c_{0} \in\left(\frac{2 a_{i}^{1}+b_{i}^{1}}{3}, \frac{a_{i}^{1}+2 b_{i}^{1}}{3}\right)
$$

such that $\nu\left(H_{c_{0}}\right)=0$ and such that $c_{0} \neq a_{i}^{j}$ and $c_{0} \neq b_{i}^{j}$ for all $j=2, \cdots, q$. This can be done because the interval mentioned above is uncountable, while $\nu\left(H_{c}\right)>0$ can occur for at most a countable number of points $c$ in the interval. We now define 


$$
R^{\prime}=\left\{x \in R_{1}: a_{i}^{1} \leqq x_{i} \leqq c_{0}\right\} \text { and } R^{\prime \prime}=\left\{x \in R_{2}: c_{0} \leqq x_{i} \leqq b_{i}^{1}\right\} .
$$

Now the $i$-mesh of $R^{\prime}$ is $c_{0}-a_{i}^{1}$ and the $i_{0}$-mesh of $R^{\prime \prime}$ is $b_{i}^{1}-c_{0}$, and both of these numbers are $\leqq \frac{2}{3}\left(b_{i}^{1}-a_{i}^{1}\right)$. It is straightforward to show that $w\left(\alpha^{\prime}\right) \subset w(\alpha) \cup H_{c_{0}}$, so that $\alpha^{\prime}$ is $\nu$-disjoint. To show that $\alpha^{\prime}$ is uneven, let $x \in I^{n}$. We must show that $p\left(\alpha^{\prime}, x\right) \leqq \xi\left(\alpha^{\prime}, x\right)+1$. If $x \in H_{c_{0}}$, then $\xi\left(\alpha^{\prime}, x\right)=\xi(\alpha, x)+1$, and it follows that

$$
p\left(\alpha^{\prime}, x\right) \leqq p(\alpha, x)+1 \leqq \xi(\alpha, x)+2=\xi\left(\alpha^{\prime}, x\right)+1 .
$$

If, on the other hand, $x \notin H_{c_{0}}$, then $x$ is not in $R^{\prime} \cap R^{\prime \prime}$, so

$$
p\left(\alpha^{\prime}, x\right)=p(\alpha, x) \leqq \xi(\alpha, x)+1 \leqq \xi\left(\alpha^{\prime}, x\right)+1 .
$$

This completes the proof of Lemma 1.

Lemma 2. Let $\alpha$ be an uneven $\nu$-disjoint rectangular cover of $I^{n}$. Fix $i \in\{0, \cdots, n-1\}$. Then there is an uneven $\nu$-disjoint rectangular refinement $\beta$ of $\alpha$ such that

$$
L_{i}(\beta) \leqq \frac{2}{3} L_{i}(\alpha) .
$$

The proof is a successive application of Lemma 1 to the members $R$ of $\alpha$.

Lemma 3. Let $\alpha$ be an uneven $\nu$-disjoint rectangular cover of $I_{n}$. Then there is an uneven $\nu$-disjoint rectangular refinement $\gamma$ of $\alpha$ such that

$$
\operatorname{mesh}(\gamma) \leqq \frac{2}{3} \operatorname{mesh}(\alpha) .
$$

The proof of Lemma 3 is a successive application of Lemma 2, starting with $i=0$, and continuing to $i=n-1$.

Proof of Theorem 1 . We let $\beta_{0}=\left\{I^{n}\right\}$, and let $\beta_{1}$ be an uneven $\nu$-disjoint rectangular refinement of $\beta_{0}$ obtained from Lemma 3. We continue applying Lemma 3 successively, obtaining $\beta_{0}<\beta_{1}<\cdots$, uneven $\nu$-disjoint rectangular covers, such that mesh $\beta_{k} \leqq\left(\frac{2}{3}\right)^{k}$. Now $\xi\left(\beta_{k}, x\right)$ is always $\leqq n$, so by unevenness, $p\left(\beta_{k}\right) \leqq n+1$. This completes the proof of Theorem 1 .

We now turn to the problem of showing that $h_{\mu}(T) \leqq h(T)$ in general.

By a representation of $(X, T)$ we mean a homomorphism from $(X, T)$ into some sequence flow $\left(B_{n}, \sigma_{n}\right)$. If $f$ is a continuous map from $X$ into $I^{n}$, we can define a representation $f^{*}$ of $(X, T)$ in $\left(B_{n}, \sigma_{n}\right)$ as follows:

$$
f^{*}(x)(n)=f\left(T^{n} x\right) \quad \text { for } n \in N, x \in X
$$


It is easily seen that all representations can be obtained this way. If $\phi$ is a representation of $(X, T)$ in $\left(B_{n}, \sigma_{n}\right)$, we write $T_{\phi}=\sigma_{n} \mid \phi(X)$, so that $\left(\phi(X), T_{\phi}\right)$ is a homomorphic image of $(X, T)$. Let $R(X, T)$ be the set of all representations of $(X, T)$. We include the following theorem for completeness, though it will not be used in the proof of our main result.

ThEOREM 2. $h(T)=\sup \left\{h\left(T_{\phi}\right): \phi \in R(X, T)\right\}$.

Proof. Let $\alpha=\left\{U_{0}, \cdots, U_{n-1}\right\}$ be an open cover of $X$. Define $f: X \rightarrow I^{n}$ as follows:

$$
f(x)_{i}=d\left(x, X-U_{i}\right) / \delta \quad \text { for } i=0, \cdots, n-1,
$$

where $\delta$ is the diameter of $X$. Let $\phi=f^{*}$. For $i=0, \cdots, n-1$, let

$$
V_{i}=\left\{y \in B_{n}: y(0)_{i}>0\right\},
$$

and let $\beta=\left\{V_{i} \cap \phi(X): i=0, \cdots, n-1\right\}$. It is clear that $\beta$ is an open cover of $\phi(X)$ and that $\phi^{-1}(\beta)=\alpha$. Hence $h(\alpha, T)=h\left(\beta, T_{\phi}\right)$ $\leqq h\left(T_{\phi}\right)$. Now since $\alpha$ was an arbitrary finite open cover of $X, h(T)$ $\leqq \sup \{h(T \phi): \phi \in R(X, T)\}$. The reverse inequality follows from general properties of topological entropy.

To prove an analogous result for measure theoretic entropy, we need the following

Proposition 5. Let $\alpha$ be a finite open cover of $X$ and let $\mu \in M(X, T)$. Then $\alpha$ has a finite closed $\mu$-disjoint refinement.

Proof. We can assume that each member of $\alpha$ is essential. Write $\alpha=\left\{U_{0}, \cdots, U_{n-1}\right\}$. Choose a closed cover $\left\{F_{0}, \cdots, F_{n-1}\right\}$ such that $F_{i} \subset U_{i}$ for $i=0, \cdots, n-1$, and let $f_{i}$ be a continuous realvalued function on $X$ which is zero on $F_{i}$ and one on $X-U_{i}$. For fixed $i \in\{0, \cdots, n-1\}$, notice that $\left\{f_{i}^{-1}(r): 0<r<1\right\}$ is an uncountable pairwise disjoint collection of closed sets of $X$, and hence the sets $f_{i}^{-1}(r)$ cannot all have positive measure. Choose $r_{i} \in(0,1)$ such that $\mu\left(f_{i}^{-1}\left(r_{i}\right)\right)=0$. Let

$$
V_{i}=\left\{x \in X: f_{i}(x)<r_{i}\right\},
$$

and let $\beta=\left\{V_{0}, \cdots, V_{n-1}\right\}$. It is clear that $\beta$ is an open cover and that $\bar{\beta}=\left\{\bar{V}_{0}, \cdots, \bar{V}_{n-1}\right\}$ is a refinement of $\alpha$. Furthermore, $\mu\left(\bar{V}_{i}-V_{i}\right)$ $=0$ for $i=0, \cdots, n-1$. We now define $G_{0}, \cdots, G_{n-1}$ as follows:

$$
G_{0}=\bar{V}_{0} ; \quad G_{1}=\bar{V}_{1}-V_{0} ; \text { in general, }
$$

$G_{i}=\bar{V}_{i}-U_{j<i} V_{j}$ for $i=0, \cdots n-1$. It is clear that $\beta=\left\{G_{0}, \cdots, G_{n-1}\right\}$ 
is a closed refinement of $\alpha$. The fact that $\beta$ is $\mu$-disjoint follows from the inclusion $G_{i} \cap G_{j} \subset \bar{V}_{i}-V_{i}$, for $j<i$. This completes the proof.

If $\phi:(X, T) \rightarrow(Y, S)$ is a homomorphism and if $\mu \in M(X, T)$, we can define a measure $\phi(\mu) \in M(Y, S)$ as follows:

$$
\phi(\mu)(A)=\mu\left(\phi^{-1}(A)\right)
$$

for every Borel set $A \subset Y$.

Theorem 3. If $\mu \in M(X, T)$, then

$$
h_{\mu}(T)=\sup \left\{h_{\phi(\mu)}\left(T_{\phi}\right): \phi \in R(X, T)\right\} .
$$

Proof. For each positive integer $m$, choose an open cover $\alpha_{m}$ of $X$ with mesh $\leqq 1 / m$. Now by Proposition 5 there is a finite closed $\mu$ disjoint refinement $\beta_{m}$ of $\alpha_{m}$. Let $\gamma_{m}=\beta_{1} \bigvee \beta_{2} \bigvee \ldots \vee \beta_{m}$. It is clear that $\gamma_{m}$ is also a finite closed $\mu$-disjoint refinement of $\alpha_{m}$. As in the proof of Corollary 1, we can show that the sigma-algebra generated by $U_{m=1}^{\infty} \gamma_{m}$ is the algebra of Borel sets of $X$. We can now apply Rohlin's theorem and obtain

$$
h_{\mu}(T)=\lim _{m \rightarrow \infty} h_{\mu}\left(\gamma_{m}, T\right) .
$$

We now fix $m$ and write $\gamma_{m}=\left\{U_{0}, \cdots, U_{n-1}\right\}$. We define $f: X \rightarrow I^{n}$ as follows:

$$
f(x)_{i}=d\left(x, U_{i}\right) / \delta \quad \text { for } i=0, \cdots, n-1,
$$

where $\delta$ is the diameter of $X$. We let $\phi=f^{*}$. Next, we let

$$
V_{i}=\left\{y \in B_{n}: y(0)_{i}=0\right\} \quad \text { for } i=0, \cdots, n-1,
$$

and $\gamma_{m}^{\prime}=\left\{V_{i} \cap \phi(X): i=0, \cdots, n-1\right\}$. It is clear that $\gamma_{m}^{\prime}$ is a closed cover of $\phi(X)$ and that $\phi^{-1}\left(\gamma_{m}^{\prime}\right)=\gamma_{m}$. It follows that $\gamma_{m}^{\prime}$ is $\phi(\mu)$-disjoint and hence we have

$$
h_{\mu}\left(\gamma_{m}, T\right)=h_{\phi(\mu)}\left(\gamma_{m}^{\prime}, T_{\phi}\right) \leqq h_{\phi(\mu)}\left(T_{\phi}\right) .
$$

We now let $m$ tend to infinity and obtain

$$
h_{\mu}(T) \leqq \sup \left\{h_{\phi(\mu)}\left(T_{\phi}\right): \phi \in R(X, T)\right\} .
$$

The reverse inequality follows from general properties of entropy.

Theorem 4. If $\mu \in M(X, T)$, then $h_{\mu}(T) \leqq h(T)$.

The proof is an application of Theorem 3 and Corollary 2.

The author would like to thank Roy L. Smith for correcting the proof of Proposition 5. 


\section{REFERENCES}

1. R. Adler, A. Konheim and M. McAndrew, Topological entropy, Trans. Amer. Math. Soc. 114 (1965), 309-319.

2. P. Billingsley, Ergodic theory and information, Wiley, New York, 1965.

3. L. W. Goodwyn, Topological entropy and expansive cascades (dissertation), University of Maryland, 1968.

4. A. I. Khinchin, Mathematical foundations of information theory, Dover, New York, 1957.

5. V. A. Rohlin, Entropy of metric automorphisms, Dokl. Akad. Nauk SSSR 124 (1959), 880-983.

6. - New progress in the theory of transformations with invariant measure, Uspehi Mat. Nauk 15 (1960), no. 4 (94), 3-26= Russian Math. Surveys 15 (1960), 1-22.

UNIVERSITY OF KENTUCKY 